\title{
Filigrane
}

Écoutes psychothérapiques

\section{De quel reste à venir la psychanalyse est-elle le nom ? Entretien avec Ghyslain Lévy}

\section{Ellen Corin}

Volume 21, numéro 2, automne 2012

URI : https://id.erudit.org/iderudit/1015200ar

DOI : https://doi.org/10.7202/1015200ar

Aller au sommaire du numéro

Éditeur(s)

Revue Santé mentale au Québec

ISSN

1192-1412 (imprimé)

1911-4656 (numérique)

Découvrir la revue

Citer ce document

Corin, E. (2012). De quel reste à venir la psychanalyse est-elle le nom ?

Entretien avec Ghyslain Lévy. Filigrane, 21(2), 85-109.

https://doi.org/10.7202/1015200ar d'utilisation que vous pouvez consulter en ligne. 


\section{De quel reste à venir la psychanalyse est-elle le nom? Entretien avec Ghyslain Lévy}

\section{Ellen Corin}

hyslain Lévy est psychanalyste, membre du Quatrième Groupe; aupara$\exists$ vant, il a été membre du Collège de psychanalystes. Monsieur Lévy a une formation médicale, en psychiatrie puis en pédopsychiatrie. Il exerce à Paris, notamment depuis de nombreuses années comme psychanalyste auprès des jeunes adultes, dans le cadre du Bureau d'aide psychologique universitaire (BAPU).

Ellen Corin : Ghyslain, tu as souhaité intituler cette entrevue «De quel reste à venir la psychanalyse est-elle le nom? » La psychanalyse comme reste, qu'est-ce que tu en dirais? Quelle serait l'importance de ce reste?

Ghyslain Lévy: Ta question est énorme!

EC: Énorme, oui. Quelle voie d'entrée voudrais-tu prendre pour l'aborder? GL: C'est une formulation que j'ai voulue complexe, en choisissant de poser la question du reste à venir. Il est très important actuellement d'avoir une pensée complexe par rapport à un simplisme conceptuel formidable, mais c'était aussi pour interroger, d'une part, qu'est-ce qu'il en est de la psychanalyse comme reste, sur quel reste vient-elle s'affirmer et, d'autre part, quel est son reste? On pourrait aborder en premier lieu la question sous l'angle de ce que la psychanalyse représente comme reste aujourd'hui, c'est-à-dire comme lieu de résistance par rapport à ce qui semble de plus en plus être des opérations sans reste: la pensée technologique, la pensée du savoir-faire, les modes de pensée où domine l'évaluatif.

EC: Où domine la transparence aussi. Où il n'y aurait aucun reste derrière la surface des choses.

GL: Une logique qui vient traiter ces restes de manière à les réduire absolument. Toute la pensée actuelle est prise justement dans une volonté d'abolir le reste, la logique du reste. Tous les processus évaluatifs, toutes les opérations 
- opération de savoir-faire, opération de pensée, opération pratique, opération des discours - visent à annuler précisément tout ce qui pour un être, reste. Et la psychanalyse, par rapport à cela, est précisément le reste qui résiste ou qui voudrait résister à ces opérations économiquement et financièrement si puissantes, qui visent à traiter tout objet comme s'il était entièrement mầtrisable, qu'il ne laissait aucune place à l'indécidable ou à quelque chose qui ne serait pas totalement réductible. C'est une question fondamentale éthiquement.

EC: Quand tu intitules ton dernier livre L'esprit d'insoumission ${ }^{1}$, ce que tu as en vue, est-ce une attitude transgressive qui serait indispensable pour pouvoir traiter de ce reste?

GL: En fait, il ne s'agit pas de mon livre: c'est un livre collectif que j'ai dirigé. Ce titre, L'esprit d'insoumission, est le résultat de toutes les rencontres du comité de lecture qui y ont conduit. Évidemment, ce titre m'importe. Surtout qu'il s'agit de réflexions et contributions autour de la pensée de Nathalie Zaltzman. Je crois que l'esprit d'insoumission vise à s'opposer, à résister à ces opérations sans reste qui me paraissent être la règle générale actuellement. C'est sûr qu'il y a quelque chose du côté de l'insoumission, mais d'une certaine manière, la psychanalyse aujourd'hui se définirait de plus en plus clairement à partir de ses opposants. Par exemple dans les dernières semaines en France, la Haute Autorité de la Santé, une émanation directe du ministère de la Santé, qui a souligné que la psychanalyse, étant non pertinente et non consensuelle, n'était pas recommandable pour le traitement de l'autisme. Il était donc question de ne plus recommander la pratique de la psychanalyse pour ces deux raisons. Or, en y réfléchissant quelques instants, je me suis dit que finalement ça définissait exactement, a contrario, ce que pouvait être la psychanalyse, c'est-à-dire non consensuelle. Évidemment et heureusement qu'elle n'est pas consensuelle. Quelle drôle d'idée de dire que la psychanalyse présente un déficit de consensualité alors que c'est ce qui la définit! Grâce aux opposants de la psychanalyse, on pourrait définir beaucoup mieux celleci comme étant à la fois impertinente et non consensuelle. Voilà les deux termes qui m’apparaissent très importants pour bien désigner quelque chose du côté de l'esprit d'insoumission.

EC: Un autre aspect de cette problématique est l'orientation fermement décidée par toute l'organisation des services de santé, de soumettre l'exercice de la psychothérapie à certaines règles. Cela inclut le projet de considérer la psychanalyse comme une psychothérapie et de chercher à l'encadrer en lui imposant un certain nombre de règles qui viennent du dehors de la psychanalyse. 
Dans ce contexte-là, quelle est la marge de jeu dont dispose la psychanalyse et quelles sont les limites sur lesquelles elle ne peut pas céder?

GL: Le risque aujourd'hui est de faire de la psychanalyse une thérapie de plus. Dans un arsenal - et ce n'est pas pour rien que j'utilise le terme d'arsenal un arsenal de traitement qui vise à faire de la souffrance humaine un autre objet à réduire et à traiter dans une maîtrise complète de ce qu'il pourrait signifier et dans une annulation du sens qu'il pourrait porter. Il y a quelque chose que la psychanalyse doit soutenir, quitte à, peut-être, renoncer à un certain nombre d'enjeux de reconnaissance. Après tout, je crois que c'est la question la plus importante, si la psychanalyse est amenée à retrouver une certaine subversivité originaire, peut-être que c'est le sens qui est le plus important aujourd'hui. La question, pour la psychanalyse aujourd'hui, n'est certainement pas dans un désir de réassimilation ou de reconnaissance éperdue à l'égard des autres formes de traitement de la souffrance que propose la société.

EC: Cela me fait penser à ce qu'écrivait Nathalie Zaltzman au sujet de la guérison psychanalytique comme travail de culture, un travail qui opère nécessairement aux niveaux individuel et collectif. Il me semble qu'une des forces et une des originalités principales de ta pensée est d'avoir noué ensemble un intérêt pour ce qui est en jeu sur la scène collective et une grande attention à ce qui concerne les psychés les plus singulières. Pourrais-tu nous parler un peu de la façon dont tu articules ce double niveau? Peut-être en partant de l'autre livre que tu as écrit récemment et qui porte ce titre tellement énigmatique, à la fois fascinant et troublant, L'ivresse $d u p^{2}$ ire $^{2}$. Tu y articules de manière stimulante et troublante ta réflexion sur le collectif et tes observations sur le plan de la clinique.

GL: On pourrait le prendre par différents bouts, mais je réfléchissais à cette question du pire actuellement et je me demandais si au fond, le pire ce n'était pas cette opération qui se voulait annulatrice du reste, qui est si importante actuellement.

EC: Sous l'égide de l'Un.

GL: Oui, sous l'égide de l'Un et sous l'égide d'une uniformisation de la pensée, de la pratique et des modes d'approche de la question de la souffrance en tant qu'elle est la condition même de la subjectivité. Sur la question de comment travailler à la fois le singulier et le collectif, c'est là une des conditions, puisqu'on ne peut plus traiter actuellement une souffrance singulière sans la réinsérer dans l'histoire de l'ensemble. Toute l'œuvre de Nathalie Zaltzman a pris ses sources dans une réflexion à partir de l'histoire des camps, de 
l'horreur des génocides qui ont marqué le $\mathrm{xx}^{\mathrm{e}}$ siècle. C'est à partir de cette réflexion qu'elle a élaboré la notion du travail de culture. Cette notion, telle qu'elle l'a conçue, a tout à fait trait aux expériences limites et concentrationnaires. Autrement dit, quelque chose d'une réalité qui porte à la fois la question de cette souffrance singulière, et de la souffrance collective et historique dans laquelle elle s'inscrit. On ne peut pas aujourd'hui écouter quelqu'un dans sa souffrance singulière sans avoir au moins à l'esprit l'ensemble dans lequel il s'inscrit.

EC: Et cette ivresse du pire? Quel serait le sens de ce mot «ivresse»? Comment cette ivresse se traduit-elle dans la clinique?

GL: J'ai travaillé beaucoup avec un certain nombre d'adolescents et de jeunes adultes, d'où la question de la surenchère dans une jouissance de la destructivité et de l'auto-destructivité. Cela m'a amené à parler de la question de l'ivresse et, dans une escalade continuelle qui vise à aller toujours plus du côté d'une énergie à abolir, d'une abolition énergétique, une forme de nirvana comme disait Freud, qui est contradictoire avec tout principe de plaisir. Il y a là comme un principe d'épuisement énergétique à travers les formes d'addiction, de boulimie...

EC: Tu parles de sujets tombés hors du monde?

GL: Tout à fait. Comme si au fond, quelque chose d'un délai, d'un différé, d'une retenue par rapport à la nécessité d'une décharge énergétique était véritablement la question la plus difficile aujourd'hui à soutenir. Et ce n'est pas pour rien que je parle de quantitatif, parce qu'il me semble aujourd'hui qu'il y a une priorité accordée au quantitatif, aussi bien dans ce qu'on a évoqué tout à l'heure des modes de prise en charge, des modes d'évaluation des opérations, que singulièrement dans les modes de résolution de la souffrance. EC: Mais n'est-on pas dans une logique paradoxale, au sens où d'un côté, c'est le règne de l'institution, de l'Un, du système qui vise à annuler les différences dans une espèce d'extension à l'infini d'un Éros qui devrait rendre toutes choses étales et produire de l'homogène; et où de l'autre côté, on assiste à une violence située du côté de la quantité et qui opère à l'état brut. Où situer le pulsionnel dans ce contexte? Je pense à Malaise dans la culture où Freud parle du refoulement ou de la transformation des forces pulsionnelles qui nous habitent, requis par les exigences du vivre ensemble et nécessaire à l'advenue d'un être de culture. Faudrait-il repenser la notion même de travail de culture dans des termes plus contemporains?

GL: C'est le travail que j'ai pu faire récemment dans L'ivresse du pire, c'est également le travail que j'ai partagé avec Nathalie Zaltzman. Un travail sur le 
fait de pouvoir penser non plus une culture au sens cumulatif des produits culturels - c'est un peu dans ce sens-là que Freud l'utilise bien souvent, l'accumulation culturelle - mais du côté de ce qui fait résistance à tout ce qui met en péril la réalité humaine, et de ce point d'irréductibilité qui viendrait signifier que quelque chose de l'humain continue à exister malgré tout. C'est cet enjeu-là du malgré tout qui semble aujourd'hui au cœur d'une écoute analytique qui assume sa radicalité. Quelque chose de ce point de résistance qui a gravement été mis en péril dans les catastrophes du $\mathrm{XX}^{\mathrm{e}}$ siècle.

EC: Nathalie Zaltzman et d'autres psychanalystes se sont beaucoup penchés sur les camps; toi tu prolonges cet intérêt pour les camps mais tu l'as élargi ou déplacé en introduisant Hiroshima et Nagasaki, qui sont d'autres catastrophes humaines de grande envergure. Tu parles dans un de tes textes récents de l'importance d'adopter une optique transtraumatique, ce que j'entends par contraste avec la fixation sur un point qui serait considéré comme une sorte d'acmé de la violence. Pourrais-tu nous en parler un peu plus? Cela me paraît important si l'on veut demeurer ouvert à ce qui se passe dans le monde contemporain, par-delà ce qui s'est passé dans les années 1940.

GL: Tout à fait. Par fixation, je voulais dire un point d'irréductibilité du trauma, qui fait effet de colle, de collage qui désubjective dans le désastre. La question du transtraumatique, je la posais plus au plan du contre-transfert. Comment entendre, écouter et accueillir une souffrance qui vient rencontrer l'extrême, et lui donner la possibilité non pas d'une abolition, d'une annulation, d'une anesthésie, ce qui serait totalement mortifère, mais de trouver avec l'autre un destinataire possible d'une parole qui puisse border quelque chose d'un réel souvent non dicible? Écouter ce type de singularité dans sa fixation même ne peut déjouer quelque chose de l'enjeu qui est là qu'à la condition que l'analyste puisse lui aussi mettre à l'œuvre quelque chose de ses propres extrêmes, de ses propres confins. De là où il a fréquenté et fréquente ces confins. Il y a là une dimension de transtraumatique qui rend le dialogue possible non pas dans l'indifférence et dans la confusion des expériences et des événements, mais dans la possibilité d'un partage.

EC: Ce qui signifie que l'analyste doit aussi faire ce chemin à rebours pour retrouver ce qui est en carence de mots, y compris en lui.

GL: C'est là aussi la singularité de travailler avec des patients qui ont été soumis à la torture, qui ont vécu des situations extrêmes, qui ont dû fuir leur pays et se retrouver dans des situations d'exil déshumanisant. C'est une clinique singulière, mais qui nous apprend beaucoup sur les autres formes de la clinique. Aujourd'hui, on ne peut pas faire l'impasse de ce type d'éclairage, 
d'incandescence, d'acmé même, pour approcher la question de la souffrance; nous avons à tenir compte des contextes très particuliers dans lesquels nous travaillons de nos jours.

EC: Tu parles aussi de l'analyste comme témoin; dans quel sens? et témoin de quoi? En effet, il n'est pas en notre pouvoir de confirmer que quelque chose a eu lieu dans la réalité, d'en confirmer la "vérité matérielle». Comment penser alors cette fonction de témoin qui permette à quelque chose de prendre place dans une parole adressée?

GL: Tu as dit exactement le mot: témoin d'une adresse, témoin que quelque chose a pu être adressé.

EC: ... ou va pouvoir être adressé alors que c'était resté sans adresse.

GL: Voilà. C'est exactement ça que je voulais dire. C'est qu'il y a quelque chose quand même qui, dans l'expérience, du moins dans l'événement qui n'est pas encore devenu une expérience, se singularise par la non-adresse, par le fait qu'il n'y a pas de destinataire à une parole quelconque qui chercherait à en dire quelque chose. Ce que le sujet vient chercher auprès de l'analyste, c'est que précisément celui-ci puisse entendre à travers l'inarticulé, le silence, le mutisme, le gel des affects... que l'analyste puisse entendre quelque chose qui lui soit adressé. Cela revient à construire une adresse, ce qui ne va pas de soi. Ça prend du temps, ça demande de créer une écoute qui permette un premier habitat possible, un premier abri à ce qui n'a pas été abrité. Construire dans l'oreille de l'analyste un premier abri fait partie de cette dimension du témoignage.

EC: Tu as parlé de la différence entre un événement qui a eu lieu et une expérience. Pourrais-tu nous en parler davantage? Comment cela nous permetil de mieux comprendre l'effet de transformation opéré par la psychanalyse? GL: Un événement n'a pas eu de lieu psychique pour exister, n'a pas d'existence pour la réalité psychique. Freud nous dit quand même que la réalité c'est d'abord la réalité psychique avant d'être la réalité matérielle ou historique. Parce qu'elles deviennent matériau psychique, la réalité matérielle et la réalité historique peuvent être prises en compte. La possibilité de fabriquer un premier abri à un événement qui n’a pas encore eu de lieu psychique pour commencer à exister permet l'expérience, soit précisément cette transformation de l'événement en matière psychique. Ce qui fait une expérience, c'est la transformation de l'événement, qui n’a pas eu de lieu, en matière psychique.

EC: Je me demande si on ne pourrait pas dire la même chose au niveau collectif. Je pense au nombre d'années qu'il a fallu avant qu'on commence à 
repenser à la question des camps d'extermination. Cela a fini par se faire, sans doute parce qu'on se sentait tous plus ou moins concernés par ce qui avait eu lieu. Par contre, ce qui s'est passé à Hiroshima et Nagasaki semble ne pas avoir trouvé de lieu psychique propre, du moins, dans notre culture. Quelque chose de cette temporalité double semble donc également en jeu sur la scène collective.

GL: C'est d'autant plus vrai que ce sont des moments de franchissement inédit par le nucléaire; le nucléaire dans sa dimension catastrophique et dans sa dimension humaine, c'est-à-dire qu'il ne s'agissait pas d'un accident.

EC: C'était planifié, voulu.

GL: Une volonté humaine d'utiliser le nucléaire ce qui fait toute la différence. Ça s'inscrit comme un événement inédit qui a ouvert l'ère nucléaire. L'ère nucléaire contemporaine s'ouvre sous le signe d'Hiroshima et de Nagasaki. Alors, peut-être que le fait qu'il n'y a pas de lieu psychique pour penser vraiment cette dimension-là n'est pas sans rapport avec tous les enjeux qui sont les nôtres aujourd'hui pour aborder la question du nucléaire, de l'économie du nucléaire dans notre monde. Actuellement, l'extrême difficulté d'avancer quelque chose du domaine politique, de réinterroger les choix économiques et donc des choix de société, qui sont faits autour de cette question: Fukushima en est l'exemple le plus évident, bien qu'il s'agisse ici d'accident. L'impossibilité de parler de l'accident de Fukushima aujourd'hui est quelque chose d'exemplaire, qui signe ce que tu dis sur le fait qu'il n'y a pas encore eu de lieu psychique pour penser le franchissement inédit du nucléaire.

EC: Là en fait, ce qui est en jeu, et tu le dis dans ton livre L'ivresse du pire, c'est une possibilité d'extermination qui porterait sur l'ensemble de l'humanité. Or, on n'arrive pas à penser cette possibilité.

GL: Voilà. Il y a le fait qu'on n'arrive pas à la penser parce qu'elle touche une dimension de l'humanité qui est en effet probablement du côté de l'impensable, de l'irreprésentable, mais je pense aussi en même temps, en t'en parlant, qu'il y a une telle conjonction avec les pouvoirs politiques et économiques aujourd'hui, que cette question, pour d'autres raisons, se trouve de plus en plus difficile à aborder. C'est vrai qu'on a du mal à penser à la surenchère qui nous tétanise d'une autre manière - ça c'est les questions politiques et collectives -, mais il y a aussi une certaine dimension traumatique qui a un effet de sidération pour la pensée. La question Hiroshima-Nagasaki et les possibles franchissements à venir ont des effets de paralysie de la pensée qui vraiment nous rendent inaccessibles ces modes de traitement par la psyché dont je parlais tout à l'heure. Comment habiter un tel lieu? Comment faire que ce lieu 
soit habitable? Peut-être que les issues aujourd'hui en passent par l'écriture, par les travaux des écrivains. Je suis très sensible à tout le travail des écrivains, notamment des écrivains japonais, sur cette question. Probablement que ça en passe par l'art, par la possibilité de transformer au moins les bords de ce trou de réel en matière psychique où l'art est convoqué de façon tout à fait fondamentale en fait, et le travail créatif autour de ce réel.

EC: C'est exactement la question que je voulais te poser. Une des voies que tu suis pour aborder ce qui est en recherche d'expérience dans cet irreprésentable, consiste à passer par les écrivains. Et tu l'as fait aussi dans la conférence que tu as donnée il y a trois jours sur Paul Celan et la question du langage poétique. On a l'impression que les écrivains, et sans doute l'art de façon plus générale, ont une fonction de «passeur d'expériences ». Grâce à eux qui, revenant d'avoir vu la Gorgone, cherchent à en dire quelque chose, le plus souvent en bégayant, pour reprendre ton expression, nous pouvons nous approcher de ces bords et de ce qui autrement nous demeurerait inaccessible.

GL: Ce que tu dis, on devrait pouvoir le dire aussi de la psychanalyse qui constitue également l'un des ponts possibles. Je pensais au livre de Coetzee Élizabeth Costello ${ }^{3}$. Il y dit à sa manière que pour ouvrir, pour faire une ouverture, pour passer de là où on est, c'est-à-dire nulle part, à ailleurs...

EC: Quelque part, peut-être?

GL : ... il suffit de construire, de bricoler un pont. C'est vraiment toute le démarche de la psychanalyse de passer de nulle part à ailleurs, mais c'est aussi toute la question de la langue, et de l'écriture. Ce pont, il faut aussi l'entendre comme un pont au-dessus de l'abîme: nous parlons toujours au-dessus de l'abîme, nous sommes toujours au bord de la question de l'abîme. C'est ce que dit Kafka dans sa nouvelle Le pont de bois où l'homme est un pont, il fait de son corps un pont tendu d'une rive à l'autre et c'est sur son dos que passent les gens. Il a la face devant l'abîme, il regarde l'abîme et les autres peuvent d'une certaine manière le franchir en aveugle pour aller de nulle part à ailleurs. Voilà une image extraordinaire de comment soutenir l'autre dans son franchissement sous condition de soutenir le regard de l'abîme. C'est exactement ce que fait Paul Celan. Dans son écriture, il va regarder l'abîme, c'està-dire convoquer Méduse, convoquer les automates, aller jusqu'à affronter Gorgô pour faire retour d'une certaine manière, et nous renvoyer à notre image humaine sur laquelle nous pourrons nous tenir debout, sous condition de soutenir ce regard-là. Mais c'est là où Kafka a une vision tout à fait radicale puisqu'il dit: au moment où l'on se retourne pour voir qui est en train 
de franchir notre dos, alors c'est la catastrophe, le pont s'effondre. Est-ce qu'on a vu déjà un pont se retourner sur soi? Quand le regard de l'abîme n'est plus soutenable et qu'on se détourne pour peut-être regarder ailleurs, le pont s'effondre. Comme si le franchissement était soutenu par «le regard», pour Kafka : ça c'est intéressant.

EC: Qu'il doive continuer à regarder l'abîme...

GL: C'est-à-dire à soutenir le regard de l'abîme, soutenir la confrontation face à face avec l'abîme, ce qui permet à l'autre, dans son dos, de tenir le coup. EC: C'est une belle définition du rôle du psychanalyste dans le monde contemporain.

GL: Du psychanalyste et de la question de l'altérité! Soutenir l'autre dans son altérité, c'est-à-dire sans que je le voie: seulement en en soutenant le poids, sous condition de faire face à l'abîme. En effet, superbe façon de dire ce qu'est la culture quand elle ose franchir ce nulle part pour aller ailleurs. Et l'abîme au-dessus duquel il faut passer, le risque que ça suppose d'aller s'écraser au fond.

EC: Il y a aussi ce mot ailleurs que tu utilises. J'ai suggéré quelque part, mais le terme ailleurs est beaucoup plus juste. Et cet ailleurs, dans son indétermination même, montre qu'on ne sera jamais arrivé. Il y aura toujours quelque part d'autre, ailleurs.

GL: Il y a un ailleurs, mais toujours par rapport à un nulle part: le «nulle part» où je suis. Le désir de l'ailleurs me vient d'une reconnaissance du nulle part où je suis. D'une certaine manière, on approche quelque chose de l'engagement dans la situation analytique. Comment s'engage-t-on dans un projet analytique? D'abord, on ne s'engage pas seul, on s'engage si l'autre s'engage: il faut qu'il y ait un co-engagement. Mais on s'engage aussi réciproquement, dans une position asymétrique, exactement comme dans le pont de Kafka. Je m'engage dans un désir de quitter le nulle part où je suis, ou de reconnaître ce nulle part, pour le désir d'un ailleurs, sous condition qu'à l'aveugle je franchisse le pont, en sachant que vous mon analyste, vous allez pendant ce temps soutenir le face à face avec l'abîme. L'abîme en moi, l'abîme en vous, l'abîme en nous.

EC: C'est peut-être une des façons d'exprimer le malaise contemporain. Tu parles de la science comme prétention à tout savoir, tu évoques l'importance de la transparence, de l'Un dans le monde actuel; on croit avoir établi des bases extrêmement solides dans la réalité mais, quand on est sur ces bases-là, on est quelque part et il n'y a pas d'ailleurs qui compte vraiment. L'ailleurs est l'ennemi. L'ailleurs est l'hostile. 
GL: Tout à fait. Là où je suis n'est plus le nulle part; on vient revendiquer «je sais où je suis » dans une espèce de certitude, d'affirmation identitaire qui fait que c'est l'autre qui est renvoyé au nulle part.

EC: Mais, ce nulle part n'est plus porteur d'un mouvement vers l'ailleurs, c'est un nulle part où l'autre choit. On parlait de la différence entre un reste et un rebut: l'autre devient un rebut, un déchet.

GL: Il devient rebut. Il n'est plus le reste qui fait résistance, qui fait kulturarbeit, qui fait travail de culture, qui fait point d'irréductible. Il est en effet du côté du déchet, du rebut, de la fécalisation du sujet dans un effondrement. Il y a une potentialité mélancolique du sujet contemporain toujours menacé de cet effondrement, dans une fécalisation de sa subjectivité. À la hauteur du grandiose du projet qui nous est proposé, à savoir cette maîtrise absolue de l'opération qui vise à traiter un humain sans reste: étrange façon de considérer l'humain!

EC: Je me demande jusqu'où doit aller le psychanalyste dans son engagement comme psychanalyste. Jusqu'où doit aller son engagement comme personne par rapport aux enjeux du monde contemporain. Il ne peut pas s'intéresser à tout, ce qui serait opposer un projet grandiose à un autre projet grandiose. Il ne peut pas s'en désintéresser non plus. Il ne peut pas l'aborder sous l'angle d'un savoir expert qui dirait à l'autre, depuis un lieu psychanalytique «hors-champ ", quelle serait sa vérité. Est-il nécessaire que le psychanalyste s'engage par rapport aux enjeux du monde contemporain? Il y a tellement de choses en changement (je pense par exemple aux nouvelles technologies de reproduction) qu'on a parfois l'impression que nos théories psychanalytiques sont en retard, telles qu'elles sont formulées généralement. Comment alors ne pas choisir d'adopter une optique sociologique qui se demanderait par exemple : voilà quelles sont les nouvelles formes de familles, quelles en sont les implications pour nous? Posé ainsi, ce genre de questionnement ne nous conduit pas très loin. Comment rester articulés sur ces enjeux et en faire une matière qui nous pousse à remettre notre pensée au travail ? sans dénier notre position de psychanalyste qui n'est ni celle du sociologue ni celle de l'anthropologue, mais en demeurant concerné par ces enjeux?

GL: C'est un peu ce qu'on est en train de dire: soutenir la singularité de notre position vertigineuse, la question de l'abîme sur lequel, autour duquel se construit toute subjectivité, ce qui est tellement en porte-à-faux, à contrecourant de ce qui nous est proposé aujourd'hui dans tous les champs - ceux de la science et des pratiques technologiques, notamment. C'est à partir de 
notre position singulière qu'on peut essayer de dire quelque chose, d'une éthique du risque, de ce qui n'est jamais clos, du reste.

EC:... d'une position singulière qui se sente en même temps interpellée par ce qui se passe autour de nous, parce que certaines positions singulières travaillent sur le strict plan individuel et ne se sentent aucunement concernées par les enjeux de la culture.

GL: Oui, évidemment. Quand je dis une position singulière, c'est par rapport à une position d'expert. Parce qu'évidemment, les analystes sont continuellement sollicités à prendre des positions d'expert et ils le font dans bien des cas. Ils se retrouvent donc dans une position de surplomb, dans une position de maîtrise. Précisément cette question de l'engagement - dans le vertige que cela suppose, dans la fabrication d'une demande, dans ce qui ne va jamais de soi me semble au plus près des questions contemporaines. On a parlé de la danse ensemble, de toutes ces formes d'expression gestuelles, du corps, dans les cultures des marges, dans les cultures que proposent actuellement les banlieues; des cultures qui ne sont pas reconnues officiellement. Je pense que la psychanalyse a peut-être à accepter une position de marge, surtout actuellement. Qu'elle a à renoncer à un désir de reconnaissance éperdue qu'elle a pu avoir à un certain moment, qu'elle peut avoir à certains moments aussi, que certaines institutions peuvent revendiquer. Il y a une éthique actuellement de psychanalystes qui font de leur singularité la condition d'un être-ensemble, ce qui me semble être un enjeu très important dans la possibilité d'être analyste aujourd'hui.

EC: Cela m'amène à un autre point que tu as développé récemment et que je trouve également fascinant: on pourrait être tenté de se placer non pas dans une position d'expert mais dans une position qui serait essentiellement dénonciatrice de tout ce qui ne fonctionne pas: l'Un, la bureaucratie, etc. Mais tu parles aussi de la métaphore des lucioles. Je voudrais que tu l'expliques un peu plus. Cela me parait important pour notre position de psychanalystes. Pourrais-tu nous en dire un peu plus sur cette question des lucioles par opposition à une vision uniformément critique de tout ce qui se passe dans le monde autour de nous?

GL: D'abord, c'est en regard de la position d'expertise : absolument intenable, impossible. Il est vrai que la psychanalyse semble être d'abord dans un vaet-vient, une alternance. J'ai parlé des lucioles, à la suite de Didi-Huberman, comme les figures même de cette posture fragile, qui s'éteint et s'allume continuellement...

EC: $\ldots$ dont le repérage dépend du regard: si on ne veut pas les trouver, on ne les trouvera pas. 
GL: Tout à fait. On parlait tout à l'heure du regard qui doit faire face à l'abîme... c'est là où on pourrait interroger Kafka: devant l'impossibilité de soutenir continuellement ce face à face avec l'abîme. Peut-être que nous avons besoin d'éclairer nos obscurités, comme disait Freud en parlant de nos obscurités intimes. Ces éclairages d'obscurités intimes ne peuvent se faire que par intermittence, alternance d'obscur et de clair. Par exemple il me semble de plus en plus que la psychanalyse a à penser et à travailler la question des bords. Quand on va voir un analyste, c'est pour essayer aussi de rendre ses propres bords fréquentables, quand ils s'avèrent absolument infréquentables. Et ces bords ne sont fréquentables que par alternance, que par ce mouvement d'éclairage en luciole. On ne peut affronter la question de fond d'un certain rapport de vérité du sujet à lui-même que par intermittence.

EC: Cela ne t'évoque-t-il pas ce que Freud écrit à propos des pseudopodes qui vont déguster de petites quantités du monde extérieur pour les digérer? C’est une image qui rejoint celle de la luciole.

GL: Tout à fait. C'est-à-dire qu'il y a une gustation, une façon de goûter l'autre, goûter l'extériorité, goûter l'altérité de l'autre qui s'appelle aussi le tact.

EC: C'est intéressant que ce mot ouvre sur ces deux sens.

GL: Les analystes qui nous ont précédés ont beaucoup travaillé cette notion de tact. On ne la réinvestit pas suffisamment: cette façon de toucher l'altérité de l'autre, mais avec subtilité, comme ce pseudopode qui vient goûter l'altérité. Je préfere parler d'altérité plutôt que d'extériorité, parce que cette image du pseudopode et de la cellule est une figure du narcissisme qui me parait un peu trop basée sur un modèle unicellulaire: un protoplasme fermé sur lui-même, défensivement confronté à l'hostilité du monde qu'il toucherait avec son pseudopode en se retirant au plus vite. On peut utiliser l'image freudienne tout à fait autrement en relevant la façon d'aller goûter l'altérité de l'autre; on n'est plus du tout alors dans cette position d'un narcissisme défensif, mais au contraire dans l'image de ce qui vient définir les conditions d'ouverture à l'altérité.

EC: Ce serait un autre aspect de la contribution de la psychanalyse au travail de culture : à travers ces palpations légères, réintroduire une attention à l'altérité interne (pas juste extérieure) aux sociétés, une altérité qui se révèlerait dans son effet de relance.

GL: Voilà, on ne serait plus dans ce dedans-dehors, dans cette espèce de dichotomie qui ne tolère pas de gradient, qui est ou dedans ou dehors, mais justement dans un espace ou une zone intermédiaire, pour prendre les termes winnicottiens. La frontière entre le dedans et le dehors ne serait plus ce mur rigide, mais elle pourrait être pensée en termes de porosité, d'un poros pos- 
sible, qui ne serait plus une aporie, qui serait la possibilité d'un passage, d'une frontière comme lieu de passage et non plus comme lieu de définition d'identité ou de l'identitaire.

EC: Je pense à ton intérêt pour les écrivains. Un des premiers livres que tu as écrit était sur un écrivain: Eugène $\mathrm{O}^{\prime} \mathrm{Neill}^{4}$ je pense?

GL: Le premier livre que j'ai écrit était sur Kafka ${ }^{5}$ !

EC: Donc, ton intérêt pour les écrivains remonte à loin. D'où te vient, comme psychanalyste, cet intérêt pour les écrivains de la limite? Tu arrives à les faire vivre et à nous interpeller dans tes écrits à partir de leur travail, d'une façon absolument remarquable. D’où te vient cet intérêt qui est tellement précieux pour nous?

GL: Probablement d'un désir de faire pont. Mon histoire personnelle est marquée par la question de faire pont entre deux continents. Il a été question de franchir et de faire qu'un franchissement soit possible. Probablement que les histoires de langue m’ont amené également à essayer de trouver des ponts. Et la littérature pour moi - pas n'importe quelle littérature -, certaines littératures qui viennent interpeller directement la question du franchissement, la question des seuils, la question des bords, la question de portes, d'ouverture et de fermeture, et de l'impossibilité de négocier autrement cette question des limites... cette littérature a été dans mon cas la condition pour approcher la psychanalyse. C'est probablement ce qui fait ma sensibilité aujourd'hui à certaines formes de psychanalyse.

EC: C'est intéressant! Ce ne serait pas la psychanalyse qui t'a amené aux écrivains, mais les écrivains qui t'ont porté vers la psychanalyse de par le creusement qu'ils introduisent dans la subjectivité et dans ses bords.

GL: Pour moi c'est tout à fait évident: c'est par le biais de certains écrivains que la psychanalyse a pu représenter à ce moment-là pour moi un enjeu aussi fort et aussi radical, que j'ai pu formuler et dans les termes que m'ont permis leur écriture.

EC: Freud lui-même prétendait que les écrivains ont des intuitions que lui met des années à construire laborieusement.

GL: En boitant !

EC: C'est pour ça que je trouve tellement important et utile, par exemple si on s'intéresse à d'autres sociétés, de voir ce que nous en disent leurs écrivains à partir de leur vision et de leur lieu propre, pour autant que l'on s'intéresse aux écrivains à la limite, dans les différents mondes.

GL: Tu as tout à fait raison. D'ailleurs, quand on parle de la question de la culture, par exemple Malaise dans la culture, il y a un vrai problème: de quelle 
culture parle-t-on? Cette question n'est jamais interrogée. Manifestement, Freud parle de la culture dominante occidentale européenne, alors que justement cette pluralité des cultures, leur diversité, leur altérisation continuelle les unes par rapport aux autres, tout cela nous interroge énormément, nous qui recevons aujourd'hui de plus en plus de gens qui viennent nous voir de tous les horizons, de tous les franchissements, de toutes les langues. Il est intéressant de remarquer par exemple comment quelqu'un vient faire son analyse dans une langue qui est la langue de l'ailleurs. On touche là pour certains analysants à des conditions sine qua non: surtout ne pas faire son analyse dans la langue de l'origine, le faire dans la langue de l'ailleurs. D'emblée la question du pont est interpellée.

EC: Cela me fait penser à certains cas cliniques lorsque ce pont étant établi - l'analyse dans une langue de l'ailleurs -, à un moment donné, des mots de la langue d'origine font leur chemin sur le pont et émergent dans la séance d'analyse. C'est le signe que quelque chose se passe.

GL: Tout à fait! Je demande bien souvent à ces analysants de me le dire dans leur langue d'origine. Je ne comprends strictement rien évidemment, mais soudain, on entend une voix qu'on n'avait jamais entendue, un timbre de voix je veux dire.

EC: Un rythme aussi.

GL: Un rythme, une respiration, un timbre, une sonorité, une vocalité sonore, une sensorialité des mots qui n'a plus rien à voir avec la voix à laquelle on est habitué. C'est une expérience unheimlich, étrangement inquiétante d'un familier qui devient tout à fait étranger. Cela aussi est en jeu dans la nécessité pour certains d'aller faire une analyse dans une langue de l'ailleurs: paradoxalement retrouver et non pas s'éloigner!

EC: Pour retrouver autrement.

GL: Voilà! C'est très important, non pas pour s'éloigner de la langue ou de ce que porte la langue de l'intime, mais pour retrouver l'intime. Peut-être un intime perdu, un intime inaccessible et qui ne peut être retrouvé que par ce chemin de l'extérieur. Toute forme de littérature, en tant qu'elle est radicale, nous permet d'aller faire le détour pour aller chercher dans le plus loin, le plus intime inaccessible. Le plus intime inaccessible c'est précisément ces moments où un patient ou une patiente se met à vous chanter dans sa langue d'origine une comptine.

EC: Réciter un poème...

GL: Un conte d'enfants, un poème, dire des mots comme ça. J'ai une patiente qui s'est mise soudain à me réciter un poème qui venait du plus lointain des 
montagnes, du lieu de résistance politique d'où elle venait, qui a plus ou moins disparu de par les violences militaires qui ont été effectuées sur ces zones. Comme si c'était le territoire survivant avec lequel elle pouvait vivre, qu'elle pouvait partager intimement avec moi et avec elle-même; un territoire de langue, un territoire de mémoire, un territoire de poèmes qui faisait retour, alors que son lieu territorial avait disparu. Comme si ces territoires de langue continuaient à exister là où le pays d'une certaine manière avait disparu ou était inaccessible.

EC: Je pense aussi à un cas, toujours dans ce même registre, où la personne me dit: «c'est ce mot, mais je ne peux pas le traduire, vous ne le comprendriez pas. Il n'y a pas d'équivalent, vous ne pouvez pas comprendre. Je peux vous en parler un peu à côté, mais ce que ça représente vous n'y avez pas accès. » Cela me fait penser à ce dont on parlait : ce qui reste secret par rapport à cet idéal de transparence. Quelque chose est montré mais « vous n’irez pas là, parce que ce n'est pas à vous ».

GL: Ça c'est vrai. J'avais parlé du désir de ne pas être compris que Piera Aulagnier avait théorisé il y a longtemps, mais il me semble que ça va plus loin que ce désir, il y a quelque chose d'une...

EC: ... d'une aura, d'une aura qui n'est pas traduisible.

GL: L'aura n'est pas traduisible, mais en même temps, c'est comme si elle était habitée pour la première fois aussi. C'est ça que je voulais dire. Ce n'est pas tant «je parle d'un pays où vous ne serez jamais invité», mais «c'est un pays où je n'ai jamais été, qui est le mien.»

EC: ... qui brusquement...

GL : ... qui brusquement surgit. Et ce «mais vous ne comprendrez pas!» a d'une certaine manière quelque chose de partagé avec celui qui l'exprime: nous sommes tous les deux sur le seuil, le bord.

EC: Ce qui nous ramène à la clinique parce que dans la psychanalyse, ce sont les mots, la parole qui importent; or j'ai l'impression que dans ce que tu nous dis, on n'est pas uniquement dans le sens des mots, un sens qui s'offrirait à l'interprétation. Il y a une sensorialité de la langue, c'est le mot que tu as utilisé, alors comment cela joue-t-il et quelle est l'importance de cette sensorialité des mots ou des rythmes dans la démarche de la clinique analytique?

GL: Comment arriver à s'autoriser à cette intimité-là?

EC: Oui...

GL: Parce qu'on ne dit pas à n'importe qui ce genre d'intimité. Et le plus intime est non pas dans les contenus mais dans la forme, c'est-à-dire par exemple dans l'accent qui veut être éradiqué et qui soudain surgit. Il faut 
beaucoup de confiance dans l'autre pour faire surgir une certaine musicalité des mots, cet accent des mots qui parlent du plus intime. Le découvrir pour l'autre, mais le découvrir aussi pour soi-même! Il y a là un partage qui justement pose la question de l'engagement. Tout dépend de la manière dont ça s'engage et dont ça continue à s'engager, à produire un engagement. C'est pour ça que je disais que la demande d'analyse n'est jamais avant nous, jamais à priori. Je suis de plus en plus convaincu à partir de mon expérience que la demande se construit, qu'il s'agit de la penser après-coup. Parfois elle devient un peu plus énonçable à la fin de l'analyse, mais jamais avant. Il s'agit peutêtre de repenser la question des temporalités: elles sont toutes différentes de notre temporalité linéaire à laquelle on est si accroché.

EC: Je pense à certaines façons de travailler en psychanalyse, où l'on évalue la demande en une ou deux séances pour ensuite décider d'un mode de réponse. Cela m’a toujours semblé aberrant parce que c'est dans le cours de ce qui va se mettre en place que quelque chose émerge. Comment le savoir au point de départ?

GL: Bien sûr. La demande se fabrique à deux. Je suis tout à fait d'accord: il y a eu une époque où l'on a pensé la psychanalyse avec les séances préliminaires, la décision, l'engagement dans le dispositif, et cetera. Souvent cette conception est associée à des idéaux, des modèles théoriques et pratiques qui nous empêchent de penser complètement; il s'agit de se libérer de ces modèles et ces idées.

EC: $: .$. qui dépendent d'une sorte de pré-grille à partir de laquelle on essaierait d'interpréter la singularité... ce qui est tout à fait paradoxal.

GL: Ce qui pose aussi la question de l'interprétation et de là où elle est élaborée, d'où elle est pensée puis énoncée. Il y a une certaine façon d'objectiver la pratique et l'interprétation analytiques sur le mode de leur application. On est alors dans la psychanalyse appliquée. La véritable psychanalyse appliquée n'est pas tant là où on la pense - une psychanalyse qui travaillerait en tissage avec l'art, l'écriture, et cetera -, elle est souvent à l'intérieur même de la pratique analytique. Elle est appliquée au sens de son objectivité, de son extériorité. Je pense au contraire que l'interprétation est d'abord surprise pour celui qui l'énonce avant même qu'elle soit surprise pour celui à laquelle elle se destine. L'analyste surpris: c'est la condition même de l'adresse d'une interprétation. Faut-il encore s'autoriser à être surpris par sa propre interprétation qui survient d'on ne sait où, avec un effet de surprise... Même la vocalité, la voix avec laquelle elle s'exprime est parfois surprenante. C'est à cette condition qu'elle a un effet d'implication, d'adresse. 
EC: Est-ce qu'on peut penser que cet intérêt que tu as pour la dimension poétique du langage dans l'analyse, la sensorialité de l'expression verbale, est aussi lié au fait que le travail de la cure ne consiste pas seulement à reconstruire une histoire mais aussi à rejoindre un pulsionnel infantile et donc, ce qui par définition est hors-histoire? Lui construire des voies de dégagement? Est-ce que tu pourrais en dire quelque chose?

GL: Ce que tu évoques, c'est la possibilité d'un repoussement vers les lointains et parfois, ce qui est plus intéressant encore, vers l'impersonnel. Cette dimension qui va non pas du singulier au plus singulier, mais du singulier au commun.

EC: Mais un commun qui ne serait pas le commun de tout à l'heure où tout le monde est pareil.

GL: Pas du tout.

EC: De quel commun s'agit-il?

GL: D'un commun-communauté, qui fait communauté humaine.

EC: ... qui est la condition humaine.

GL: Oui et ce rebroussement vers le plus impersonnel, vers ce qui dans la langue est porté du côté de l'incondition, de sa fragilité. De ce rebroussement même vers tout ce qui nous parle des anciens, des ruines, de ce passé commun souvent inaccessible, mais qui donne cette direction. Il y a quelque chose d'extraordinaire, par l'aventure analytique, d'aller toucher ces zones-là. Freud nous dit que la mémoire du sujet singulier va jusqu'aux mémoires collectives les plus profondes, les plus anciennes. Il dit même que ça touche les zones d'une mémoire glacière de l'humanité; il n'hésite pas à aller jusque là. C'est extraordinaire qu'il ait pu penser que la mémoire des individus pouvait trouver ses ombilics en direction des zones les plus anciennes de l'humanité, de la planète elle-même. D'aller chercher du côté du minéral, dans Au-delà du principe de plaisir, mais aussi du côté de l'histoire glacière de notre planète. Quand Freud dit à la fin de sa vie cette formule extraordinaire: "psyché est étendue, ne le sait pas ", ça veut dire qu'elle est étendue dans toutes les directions. La psyché singulière ne le sait pas, elle a cette méconnaissance d'ellemême, cette non-connaissance de ses propres directions dans le temps, dans l'espace, dans la psyché des autres. C'est ce qui fait qu'aller chercher dans la littérature veut dire aller chercher dans les psychés autres, qui nous voisinent, l'extension, l'étendue d'une psyché qui traverse : la psyché commune, du côté de ces espaces ou de ces temps sans limite puisqu'anhistoriques, mais aussi au-delà des frontières puisque dans la psyché des autres. Tu parlais des pseudopodes tout à l'heure, mais ça va même plus loin : on est largement au-delà 
de cette figure de la cellule et de ses pseudopodes. Psyché est étendue, ne le sait pas... cette étendue sans limite et sans sa propre autoreprésentation a quelque chose de tout à fait vertigineux qui me semble au cœur même de l'expérience analytique.

EC: C'est vertigineux, passionnant et dangereux. Cela m'amène à cette dimension universelle de l'humain dont parle la psychanalyse. Tu dis en fait qu'elle va plus loin que l'humain puisqu'elle remonte jusqu'aux couches glacières, mais il y a quand même, plus proche de nous, une étendue qui court à travers les cultures. Tu en as parlé, on a de plus en plus des patients de toutes les cultures. Ainsi, qu'est-ce que cet universel qui nous fait humain, qui est la condition humaine? Comment penser, ou quel est l'intérêt pour un psychanalyste de s'interroger sur, la façon dont cette universalité se trouve mise au travail de façon différente ailleurs? Et est-ce que ce détour, avec l'ascèse que cela implique de ne pas savoir d'avance (par exemple, comment la pulsion de mort va être mise en œuvre dans un autre contexte), est-ce que cela ne peut ou ne doit pas avoir un effet en retour sur notre manière, dans notre société, de penser comment le pulsionnel travaille? J'ai l'impression qu'on tend peutêtre trop facilement à penser cette universalité en termes de notre occidentalité qui sait comment vont les choses... Comment à la fois ne pas lâcher la dimension de l'universel mais en même temps, ne pas être aveugle à notre point aveugle? Quel est l'intérêt, ou l'absence d'intérêt, de cette question par rapport à la pratique analytique de tous les jours?

GL: C'est absolument incontournable dans la pratique quotidienne. Comme on le disait, on a affaire à des patients avec lesquels il faut bien se confronter à ces altérités-là et les réduire trop vite à des histoires connues...

EC: Nos théories étant des histoires connues.

GL: Oui, c'est ça que je veux dire. À des histoires connues, à quelque chose d'une violence qui répète précisément ce qu'ils ont subi. Je suis tout à fait convaincu que de plus en plus aujourd'hui le travail de l'analyse, loin d'aller des formes de l'inconnu et de l'obscur aux formes les plus éclairées et connues - où l'on trouve évidemment toute une série de rationalisations -, nous mène dans le sens inverse du plus connu, du plus habituel, de ce qui enferme le plus (parce que justement du côté du même, de l'alter ego) à l'inconnu, l'obscur, l'irréductible et l'étranger. Aller fréquenter continuellement ces zones, ces bords-là, et avoir la possibilité de les habiter est le propre de l'expérience analytique qui va dans le sens contraire de tout ce qu'on impose aujourd'hui : la transparence, la mise à plat, la maîtrise, l'éclairage qui ne connaitrait pas son obscurité, la virtualisation généralisée. Nous sommes à une époque de mon- 
dialisation. La question des cultures qu'on essaie de soutenir est complétement écrasée par une certaine conception de la mondialisation: la neutralisation des différences, un Éros qui supprimerait toutes les déliaisons possibles. Il y a un sens très radical de la psychanalyse qui est précisément de travailler du côté de la déliaison, de cette étrangeté-là, de cette confrontation à des pluriels, à des pluralités, à des obscurités intimes diverses. Toutes les cultures portent leur singularité, mais elles ont quand même en commun - et là je reviens à ta première question sur l'humaine condition - la nécessité d'une invisibilité, d'une obscurité, d'un retrait, d'un retirement. Au fond, la nécessité que tout ne soit pas dit, que tout ne soit pas mis à plat. Toute forme de culture partage cette exigence qui aurait peut-être affaire avec l'humaine condition. Peut-être qu'on pourrait trouver là des ponts.

EC: Absolument. Ce qui serait tout à fait différent d'une pensée de la diversité culturelle en termes de logique identitaire. On n'est absolument pas là; on est dans l'opposé.

GL: Voilà. On est dans ce commun-là, qui n'est plus du tout une mondialisation ou une indifférenciation, mais plutôt une façon de faire de nos singularités un ensemble. Mais il y a quelque chose qui va plus loin dans la possibilité de soutenir pour chaque culture cette exigence qui est celle de l'humain: cette épaisseur, cette non-visibilité qui ne se donne pas d'emblée.

EC: Ceci m'amène à te poser une question. Comment dans le contexte dont nous venons de parler, repenser la fameuse phrase de Freud que Nathalie Zaltzman reprend souvent: "Là où était le Ça, le Je doit advenir. » Cela reste en partie une énigme. Comment travailler à partir de cette phrase, en reconnaissant cette place du Je mais sans penser non plus que ce Je serait détenteur d'un savoir enfin acquis sur ce qui nous échappe? Comment penser cela autrement?

GL: Là où était le Ça, du Je doit advenir, du Moi doit advenir, c'est exactement la même question que: de quelle culture on parle dans Malaise dans la culture? Est-ce que ce n'est pas d'un moi souverain, un moi dominateur, un moi impérial ou impérieux? D’une culture qui domine au nom de sa rationalité? Freud parle quand même de la dictature de la raison. Certes, il l'entend par rapport aux folies d'une pulsionnalité déchaînée et destructrice. Heureusement qu'il y a une dictature de la raison dans un monde de déchaînement pulsionnel sans limite. Il y avait là le pressentiment chez Freud de ce qui allait se passer. Mais il s'agit aussi de ramener la question de la culture, de la raison, de la rationalité des Lumières, de l'universel à une certaine forme de souveraineté, à la limite de l'idéologie. On est là aussi à la frontière. Quid de la 
psychanalyse, quid de l'idéologie psychanalytique? On est également sur des bords.

EC: On peut se demander si le Moi dont il est question n'est pas justement cette prise de conscience de la fragilité et de ce quelque chose qui échappe. Non du côté de la maîtrise mais du côté de la prise de conscience qu'on n'est justement pas dans la maîtrise. Ce serait là le travail à faire en fin de cure: on n'arrive pas en dominateur à la fin d'une cure analytique, certainement pas. GL: Certainement pas. La fin de cure participe de cette rencontre avec le renoncement à une idéalisation des figures qui ont été dominatrices. Peutêtre des figures un peu massives de la raison, du père idéal également. Il faudrait peut-être revenir sur une certaine forme de désidéalisation nécessaire, de repulsionnalisation de la question du travail de culture. Quand Freud fait de la Geistigkeit, de la vie de l'esprit l'acmé d'une raison dépulsionnalisée, l'assèchement du Zwidersee, on est dans une représentation de la fin d'une cure qui maintient une certaine forme de l'idéalisation. Pour moi, la fin de la cure participe précisément d'un réinvestissement de la désidéalisation, de la repulsionnalisation des figures.

EC: ... de la langue.

GL: De la langue. D’une certaine déphallicisation des figures idéalisées. Je pense qu'approcher la question de la fin de la cure participe précisément de ce travail de renoncement, oui.

EC: Revenons à la culture. À la lumière de ce que tu viens d'évoquer, on se trouve quand même dans un drôle de contexte. On a l'impression d'une libération d'un pulsionnel de fond - la sexualité tout azimut, la destructivité, la violence-, mais en même temps, on est sous la domination d'un Éros qui tend à homogénéiser, à aplanir, là où règne le Un, tu nous l'as rappelé maintes fois. Alors, où en sommes-nous? Comment repenser la question du pulsionnel dans le monde contemporain et comment repenser le travail de culture dans ce contexte? Quel en est l'enjeu? On parle de la massification, mais on observe à la fois une massification des échanges et un déchaînement du pulsionnel. Quelle serait la voie qui sauverait la psychanalyse dans ce contexte-là?

GL: Du côté de la résistance certainement. Mais on utilise peut-être un peu trop vite le terme de pulsionnel. Le pulsionnel c'est aussi, comme dit Freud, cette zone intermédiaire qui fait intervenir la psyché.

EC: Qui est exigence de travail pour la psyché à partir du corps. Tu as raison. GL: Freud dit bien que la pulsion est un être hybride entre les dimensions du corps, du somatique, et celle de la psyché. C'est de toute autre chose dont il s'agit par rapport à la destructivité, à la destruction... à la mort. Est-ce qu'on 
est dans le registre du pulsionnel? Si oui, on serait déjà dans une certaine forme de psychisation. Je me demande si on n'est pas du côté d'un au-delà ou d'un en-deçà du pulsionnel, puisque le pulsionnel est du côté du psychique. Il me semble que Freud a cette fantastique intuition que la pulsion de mort contiendrait dans son propre mouvement quelque chose d'une radicalité de la mort, ou de la pulsion de mort pour la mort, comme le disait Nathalie. Quelque chose qui d'une certaine manière échapperait (et ça serait peut-être le point le plus irréductible) à toutes possibilités de psychiser. Ou alors il s'agirait de penser un effet de la pulsion de mort comme un au-delà du principe psychique, mais est-ce qu'il s'agit encore d'un mouvement de la pulsion? Ça demeure ouvert comme question! Mais le terme de pulsionnalité engage quand même quelque chose de la vie psychique.

EC: Cette distinction que tu introduis me paraît extrêmement importante lorsqu'il s'agit de penser le monde contemporain.

GL: Oui! Dans le monde contemporain, on n'est peut-être pas exactement dans le pulsionnel, c'est ça que je veux dire. J'ai aussi beaucoup parlé du monde de la virtualisation. Comment est-elle investie par le pulsionnel? Évidemment que le virtuel n'est pas le fantasmatique; comment le situer? Ce n'est pas l'actuel non plus.

EC: En effet, le virtuel n'est pas le fantasmatique. Mais ne peut-on pas imaginer, et il me semble qu'on le voit dans la clinique, des patients qui utilisent le virtuel au service d'une fantasmatisation?

GL: Tout à fait.

EC: On peut donc dire que le virtuel comporte deux dimensions: l'une qui tend vers quelque chose de déshumanisant, et l'autre qui soutient des jeux possibles dans le domaine de l'imaginaire et du fantasme.

GL: Oui. Le réinvestissement par le fantasme du registre de la réalité virtuelle. Ou la virtualisation de la réalité, dans le meilleur des cas.

EC: Effectivement, dans le meilleur des cas, et ce n'est pas toujours le cas.

GL: C'est vrai. Un excès du virtuel dévitalise tout recours à la possibilité d'une repulsionnalisation fantasmatique de l'objet. Il y a certainement dans la virtualité ou dans la virtualisation de la réalité quelque chose de singulier qu'on ne connaît pas encore suffisamment, mais qui semble porter atteinte à quelque chose de fondamental dans le rapport du sujet à la réalité du monde, à soi, à l'autre, et à sa vie fantasmatique.

EC: Je me demande en t'écoutant si ce que tu as dit du décalage de la sexualité par rapport à la pulsion, et ce que tu viens de dire également par rapport au virtuel, ne peut pas aussi s'appliquer à ce qui se passe en regard de la mort. 
On a l'impression que de nos jours et dans nos sociétés, la mort est largement niée. On n'a plus à notre disposition ces longs rituels de l'après-mort où l'on apprivoisait la perte, où l'on enveloppait le corps, à travers lesquels on ouvrait au défunt la voie vers un autre monde. On assiste à une négation de la mort en même temps que s'impose une présence obscène de la mort sur nos écrans. J'ai l'impression qu'on assiste ici à quelque chose d'un peu similaire à ce dont nous venons de parler et dont on mesure encore très mal l'impact au niveau psychique.

GL: Je suis tout à fait d'accord avec toi. Probablement que cela s'origine dans cette virtualisation de la réalité. Le rapport à la mort est en train de changer et il y a une déritualisation aussi de notre vie quotidienne et de la vie collective. Mais c'est une question que j'aimerais te poser à toi qui es à l'œuvre dans ce domaine-là : qu'en est-il d'une société qui se déritualise complètement? Qu'en est-il de la fonction du rituel pour venir faire bord à une activité collective où le mythe devient grandissant, dévorateur? Un mythe qui n'est plus organisateur pour dire quelque chose de l'origine et de l'ultime, mais dévorateur et négateur du sujet humain? Une société qui se déritualiserait ne pourrait plus se protéger de certains de ses mythes pathogènes, mortifères? C'est une question que j'aimerais te poser.

EC: Je pense en t'écoutant qu'il y a peut-être là une certaine impasse dans la manière dont Freud a abordé le rituel - malgré tout l'intérêt de son travail sur ce sujet - lorsqu'il l'aborde essentiellement sous l'angle de la névrose-obsessionnelle. Or, on peut aussi considérer que le rituel met en acte un mode d'élaboration psychique qui opère par la pragmatique, par une praxis qui implique le corps et qui répond aux grandes questions qui se posent à l'humain: les rapports entre les sexes et les générations, les rythmes du cycle de vie, le rapport à la mort et la relation à l'au-delà, ce qu'il en est du sens de la vie et du mal dans la société et dans la nature. Il y a dans ces rituels quelque chose qui participe intrinsèquement à un travail de culture qui rejoint au plus près ce dont nous traitons en psychanalyse. Cette perspective contraste avec un autre mode de lecture du rituel qui repose sur une mise à distance, une altérisation de l'autre qui ne nous ouvre plus sur un enrichissement au contact d'autres modes d'élaboration de ce qu'on travaille en psychanalyse et qui le met à l'écart. L'étranger dans sa portée questionnante, se voit transformé en rebut, en quelque chose de dépassé qui ne nous interpelle pas. Quand une société perd ses rituels, quelque chose de l'humain et du tragique dans l'humain - parce que c'est toujours du tragique dont parlent les rituels sous un angle ou sous un autre - qui est fameusement mis à mal. 
GL: Mais au fond, est-ce que le rituel ne vient pas anticiper le mythe? Ne vient-il pas le préparer? C’est peut-être là la difficulté pour notre société actuelle à tolérer le rite : est-ce que le rite ne serait pas toujours quelque chose de l'émanation d'un incompréhensible? Est-ce que le rite ne vient pas toujours comme un asensé, quelque chose qui n'a pas de sens, qui est d'abord agi, un acte avant même d'être un sens, une signification, un fantasme ou un mythe? Il est d'abord un geste, un événement du corps collectif, mais qui n'est pas inscrit préalablement dans du sens, qui probablement s'ombiliquerait du côté de l'impensable, et qui serait ensuite repris par le mythe. Le mythe serait issu de la nécessité de repenser ou rendre habitable la sauvagerie asensée du rite. À ce titre, si nos sociétés sont si intolérantes aux rites, est-ce qu'elles ne sont pas intolérantes précisément à cet incompréhensible du rite, à son caractère irréductible et asensé, à ce qui du rite ferait notre noyau le plus anhistorique, irréductible, hors sens? C'est une question que je te pose. EC: C'est une question complexe et deux pistes de réflexion me viennent à l'esprit. D'une part, j'ai envie de te répondre que oui, pour autant que l'on pense cette origine non pas en termes historiques mais à la façon dont la psychanalyse parle de l'originaire comme de quelque chose qui est continuellement au travail en nous. Sous cet angle, on peut considérer que le mythe va plus loin que le rite et le prolonge, en élaborant dans le langage ce qui demeure insu dans le rituel.

GL: Oui, c'est ça que je voulais dire.

EC: D’un autre côté, on peut aussi penser que les formes concrètes que prennent les rites actualisent dans le présent quelque chose qui a été mis en scène par le mythe, selon des modalités qui varient en fonction des cultures. Sous cet aspect, le rituel s'inscrit dans un ensemble de représentations et de pratiques, il met en scène une série de récits et de symboles propres à une certaine culture, à une certaine histoire et qui marquent les formes spécifiques que revêt le rituel dans un certain contexte.

GL: Donc, tu ferais un mouvement dialectique entre les deux. La question d'un rite qui ne s'origine pas dans un tel sens, dans une signification, mais dans une sauvagerie m'a interpellé. Après tout, au début est l'acte et non le verbe. Au début n'est pas le sens, au début est l'acte. Au début est le rite, dans le meilleur des cas. Au début est la destruction, puis vient le rite pour en neutraliser la potentialité folle, mais au début probablement est le crime, le meurtre, ou même plus. Nathalie Zaltzman dit: au début est la destruction puis vient la législation qui la transforme en meurtre. C'est dire que la destruction n'est pas le meurtre. C'est dans un deuxième temps qu'il est possible de 
qualifier cette destruction de meurtre, et c'est l'acte humain de la loi qui permet de transformer la destruction en meurtre, d'en légiférer quelque chose, de la sanctionner, de désigner un châtiment, et cetera. On peut dire aussi que la loi procède du rite; Kafka en a fait toute sa littérature de cette ritualité de la loi. C'est aussi une forme de ritualité que d'aménager des issues corporelles ou des gestes qui permettent de neutraliser quelque chose d'une sauvagerie originaire.

EC: Je suis absolument d'accord avec toi qu'il y a un archaïque, un archè au meurtre qui ne se traite pas. L'idée de meurtre me paraît déjà une première mise en forme de quelque chose de plus sombre ou de plus radical, plus proche d'une pulsion de mort qui serait pure déliaison.

GL: Et cet archè, qu'on l'appelle destruction ou sauvagerie, interroge nos sociétés sans en faire une régression. Il ne s'agit pas de dire qu'on revient en arrière, il s'agit de dire qu'on crée du nouveau, une nouvelle scène à laquelle la sauvagerie est convoquée. Mais on peut en effet créer une nouvelle scène au cœur de laquelle se retrouve le rebroussement à la sauvagerie. Là je crois qu'on interpelle la question de nos sociétés contemporaines.

EC: En effet. On pourrait en rester là pour le moment. Merci Ghyslain pour cette très riche entrevue et surtout pour la richesse de ta pensée qui nous apporte tellement, à nous comme psychanalystes mais aussi à la société. Un véritable travail de culture! Alors un très grand merci.

GL: Merci pour ton accueil.

\section{Notes}

1. Ghyslain Lévy (dir.), L'esprit d’insoumission - Réflexions autour de la pensée de Nathalie Zaltzman, Paris, Campagne Première, 2011.

2. Ghyslain Lévy, L'ivresse du pire, Paris, Campagne première, 2010.

3. J. M. Coetzee, 2003, Elizabeth Costello, London, Secker \& Warburg, 2003.

4. Ghyslain Lévy, Eugène O'Neill ou l'inconvenance de vivre, Paris, Anthropos, 1994.

5. Ghyslain Lévy et Serge Sabinus, Kafka, le corps dans la tête, Paris, Scarabée, 1983.

\section{Principales publications de Ghyslain Lévy}

2011, (dir.) L'esprit d'insoumission, Paris, Campagne Première.

2010, L'ivresse du pire, Paris, Campagne Première.

2004, (sous la dir. De Kostas Nassikas) Le trauma entre création et destruction, Paris, L'Harmattan.

2002, (sous la dir. de François Richard), Le travail du psychanalyste en psychothérapie, Paris, Dunod.

2000, Au-delà du Malaise. Psychanalyse et barbaries, Toulouse, Érès. 1996, L'invention psychanalytique du temps, Paris, L'Harmattan. 1994, Eugène O'Neill ou l'inconvenance de vivre, Paris, Anthropos. 
1989, La pratique de l'analyste, Paris, Retz.

1983, Kafka. Le corps dans la tête, Paris, Scarabée.

1976, (sous la dir. de Jacques Mousseau et Pierre-François Moreau), L'inconscient. De Freud aux techniques de groupe, Paris, Retz. 